Article

\title{
Surface Morphology and Histopathological Aspects of Metallic Used Cardiovascular CoCr Stents
}

\author{
George-Razvan Pandelea-Dobrovicescu ${ }^{1,2}$, Mariana Prodana ${ }^{1}$, Florentina Golgovici ${ }^{1, *(\mathbb{D} \text {, }}$ \\ Daniela Ionita ${ }^{1,3}$, Maria Sajin ${ }^{2}$ and Ioana Demetrescu ${ }^{1,4}$ \\ 1 Department of General Chemistry, Faculty of Applied Chemistry and Materials Science, \\ University Politehnica of Bucharest, Spl. Independentei 313, 060042 Bucharest, Romania; \\ pandelea_razvan@yahoo.com (G.-R.P.-D.); mariana.prodana@upb.ro (M.P.); daniela.ionita@upb.ro (D.I.); \\ i_demetrescu@chim.upb.ro or ioana_demetrescu@yahoo.com (I.D.) \\ 2 "Carol Davila" University of Medicine and Pharmacy, 37 Dionisie Lupu Street, 020021 Bucharest, Romania; \\ maria_sajin@yahoo.com \\ 3 Faculty of Medical Engineering, University Politehnica of Bucharest, Spl. Independentei 313, \\ 060042 Bucharest, Romania \\ 4 Academy of Romanian Scientists, 3 Ilfov, 050044 Bucharest, Romania \\ * Correspondence: florentina.golgovici@upb.ro; Tel.: +40-21-402-3930
}

Received: 24 July 2020; Accepted: 18 August 2020; Published: 19 August 2020

\begin{abstract}
In this study, CoCr cardiovascular metallic stents with the same chemical composition as well as the adjacent tissues were analyzed. Recovered stents and adjacent tissues were characterized by surface morphology techniques like light microscopy (LM), scanning electron microscopy coupled with energy dispersive X-ray spectroscopy (SEM-EDS) and transmission electron microscopy (TEM). The presence of metals in the tissue from around explanted stent was also confirmed by TEM method. The released metal ions were measured using inductively coupled plasma mass spectrometry (ICP-MS). Histopathological analysis was used to highlight host tissue response to the implanted stents. EDX analysis revealed that 21 stents had the same composition. The quantities of $\mathrm{Cr}$ ions detected in the tissues were between 5 and $14 \mathrm{ppb}$ and for Co ions were between 4 and $10 \mathrm{ppb}$. The tissue sections near the layers that generated large quantities of ions showed restenosis development and the deposits of surface calcifications were highlighted. The heavy metal levels observed in this study due to corrosion are too low to cause a systemic toxic response.
\end{abstract}

Keywords: CoCr metallic stent; surface analysis; ion release; histopathological analysis; microcalcification

\section{Introduction}

With excellent biocompatibility and good mechanical and corrosion resistance cobalt-chromium (CoCr) alloys with various compositions-uncoated [1,2] and coated [3-5] - have been widely investigated for use as implant metallic materials in orthopedic, dental-and most recently in cardiovascular applications. During angioplasty [6] - but also for biliary or esophageal damage as well-a stent is implanted inside an artery blockage. Since the role of the stent is to keep a structure open, initially polymeric materials were proposed [7], but today, the most commonly used are metallic stents used for vessel occlusion in interventional therapy made from materials as stainless steel, Co-Cr alloy [8] and Ti alloy [9]. CoCr and Ti alloys well known for their applications as biomaterials are used especially for their corrosion resistance [10-13]. Ni-Ti alloy, nitinol, has been utilized as a self-expanding stent due to its shape memory and super elasticity properties [14,15]. Bioresorbable magnesium alloys are considered attractive due to its mechanical performance and potential antithrombotic properties [16]. Due to the fact that the biodegradable stents disappear within a limited time after performing their duty and chance of vessel restoration will be achieved, patients will no longer must worry about late 
stent thrombosis and avoid second surgical intervention to remove the implant $[17,18]$. Nowadays, metallic stents and especially CoCr alloys with drug encapsulated are the most promoted [19].

Using stents led to reducing the re-stenosis after surgical intervention. An actual problem using stents is their fracturing, detected on one percent to three percent coronary stents and $37 \%$ of peripheral stents $[20,21]$. One of causing factors leading to stents fracturing is the electrochemical corrosion process [22]. Taking into consideration the high number of stents annually implanted, it is very important to study the processes that lead to their fracturing. Metallic stents being made of corrosion resistant alloys, develop on their surface layers of oxides when get in contact with oxygen [23]. After destruction of oxide layer, the alloy enters in direct contact with physiological fluid and may suffer electrochemical corrosion. The use of metallic stents was found to be associated with in-stent restenosis, a multifactorial process consisting of inflammation and neointimal tissue hyperplasia. Introducing surface modification as drug-eluting stents is a way to reduce the stent corrosion and to minimize restenosis complications [24,25].

The reducing of the restenosis process is based on the ability of the drug-eluting stents to inhibit the proliferation of smooth muscle cells in arterial media and the platelet adhesion, activation and aggregation. The retardation of endothelialization on stent surfaces is another possible complication and the development of surface modifications can accelerate endothelialization on stent surfaces changing drastically wettability and decreasing platelet adhesion [26] and its inhibitory effect on platelet activation [27].

The fineness of a stent can affect its performance and biocompatibility. Smooth surface can reduce thrombus adhesion and increase neoendothelium. To obtain smoothness, the stent must be treated with pickling acid and then electrochemical polishing. The process removes slag that includes deposits and burrs, formed on the surface of the stents due to the production process, by laser cutting.

To better understand the importance of stent surfaces and stent-tissue interface, the present manuscript proposes the evaluation of coronary stents recovered during autopsy or vascular surgery by microscopic investigation of stent surfaces and stent-tissue interface, histopathological and immunohistochemistry analysis of surrounding tissues. Such investigations of stent surface and tissue/stents interface at microscopic level including ion release in surrounding tissue clearly bring information about stent behavior in their exploitation time and to our best knowledge is a part of this paper novelty. The manuscript completed with histopathological and immunohistochemistry analysis of surrounding tissue could be useful in developing more knowledge about stents.

\section{Materials and Methods}

The 35 inserted stents (P1-P35) recovered during human autopsy or cardiovascular surgery were Presillion Plus type purchased from Cordis Company, having the following chemical composition Co $50.5 \%$, Cr 24.2\%, Mo 6\%, Mn 0.3\%, V 0.45\%, Ti 4.82\%, C 2.2\%, O $11.1 \%$ and Al 0.7\%. Surfaces of the stents were processed following the surface preparation guidelines described in ASTM F-86-01 [28]. All metallic CoCr stents were introduced into human bodies at about the same time and for the same period.

To evaluate the recovered stents and the nearby coronarian and myocardial tissues the following analysis were performed: surface morphology, metallic ion release quantification and histopathological evaluation. During autopsy, coronarian and myocardial tissue were also collected. Considering the stages-but also the diversity of methods used for evaluation in this study - we have elaborated a schematic research design for the investigated metallic used cardiovascular $\mathrm{CoCr}$ stent as well as for the adjacent tissues, shown in Figure 1. 


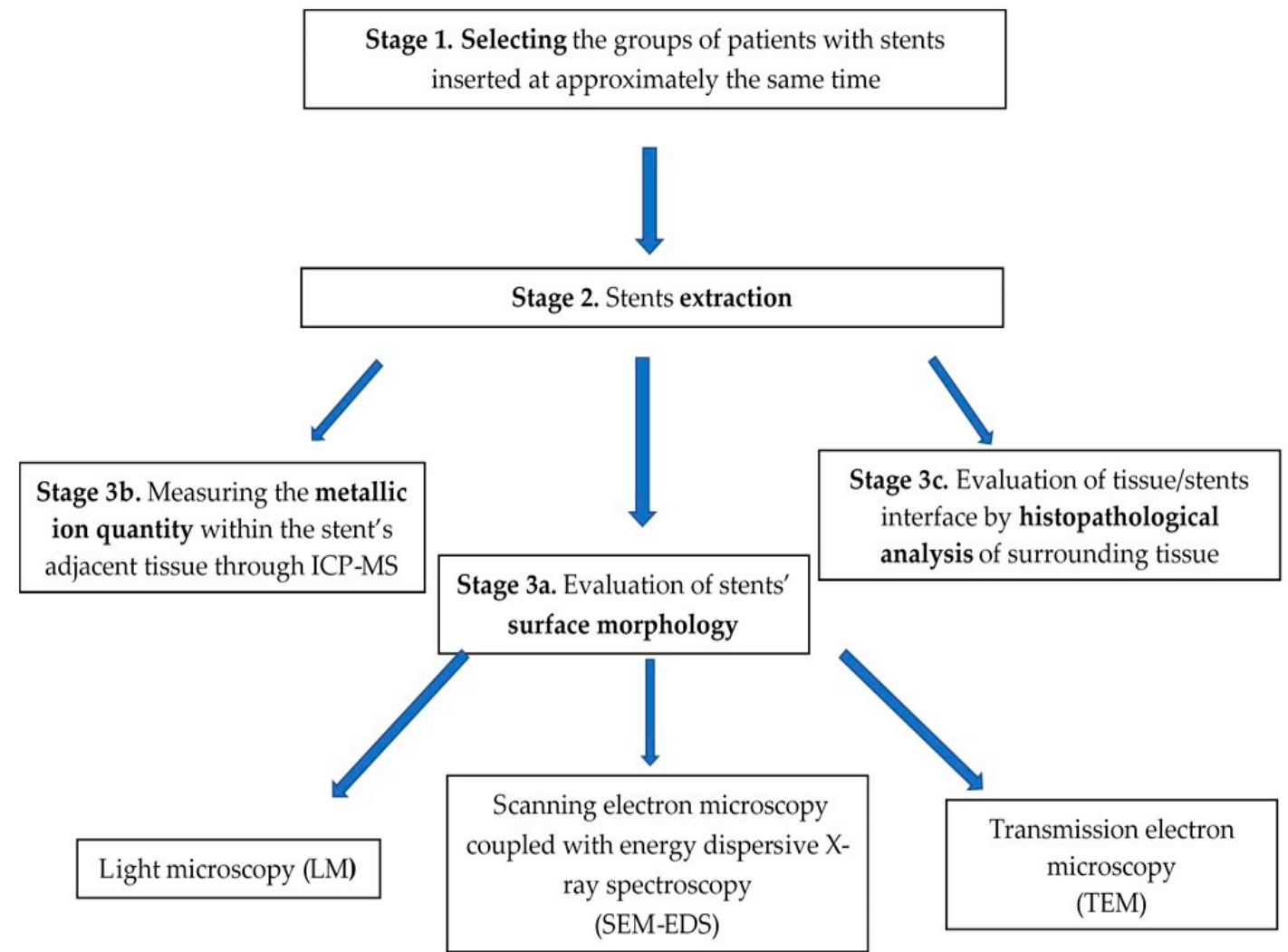

Figure 1. Schematic representation of research design.

Tissue samples were fixed with $10 \%$ buffered formalin and were processed using conventional histopathological methods used in Pathology routine, featuring inclusion in paraffin and hematoxylin-eosin (HE) staining. The resulting slides were examined in bright field microscopy and pictures were taken using Leica DM750 microscope with ICC50 HD camera (MEDIST Life Science, Bucharest, Romania).

The study was conducted in accordance with the Declaration of Helsinki and the protocol was approved by the Ethics Committee of "Carol Davila" University of Medicine and Pharmacy Bucharest, Romania, no. 75/15.10.2015 (Project identification code PO-35-F-03).

2.1. Post Exploratory Evaluation of Stents' Surface through Light Microscopy (LM), Scanning Electron Microscopy Coupled with Energy Dispersive X-ray Spectroscopy (SEM-EDS) and Transmission Electron Microscopy (TEM)

Light microscopy was performed using a Zeiss Axio Scope A1 optical microscope (Boston Microscopes, Wilmington, MA, USA) with a maximum magnification of $100 \times$.

The morphology of the stents was studied by SEM model Quanta 650 FEG from FEI company (Hillsboro, OR, USA) at a pressure of 0.7 torr at $15 \mathrm{kV}$. For the SEM measurements it was used a secondary electron detector, SE. EDS analyses were performed to identify the elemental composition of the samples. Nanosized particles were investigated using an EM-410 Phillips transmission electron microscope (Phillips, Eindhoven, The Netherlands) at $60 \mathrm{kV}$.

\subsection{Measuring the Metallic Ion Quantity within the Stent's Adjacent Tissue through ICP-MS}

Ion concentrations were quantified using inductively coupled plasma mass spectrometry (ICP-MS), work that was carried out under constant temperature and humidity conditions, ensured by working in an atmosphere free from contaminants of clean class ISO class 7 enclosures. The ICP-MS equipment an ELAN DRC-e inductively plasma mass spectrometer (Perkin Elmer, Waltham, MA, USA) and 
measurements conditions (including digestion with concentrated nitric acid and dilution) were described in previous papers. The liquid fractions were analyzed and detection limit is $0.001 \mu \mathrm{g} \cdot \mathrm{g}^{-1}[29,30]$.

\subsection{Evaluation of Tissue/Stents Interface by Histopathological Analysis of Surrounding Tissue}

In the morphologic study of tissue was used the classic histological technique by paraffin including, which involved the following times: fixation of the pieces in 10\%-tamponated formol solution for $24-48 \mathrm{~h}$, depending on the size and nature of the work piece; removal of excess formol by washing in tap water for 8-12 h; dehydration, using successively four baths of alcohol with increasing concentrations. The pieces were kept in each bath for $3 \mathrm{~h}$; clarification, passing successively the dehydrated pieces by 3 xylol baths; the duration per each bath being one hour; they were coated with paraffin by successively passing the thermostatic parts of the clarified pieces through three melted glass wafers at $56^{\circ} \mathrm{C}$. The duration of the baths was six hours per bath; inclusion of paraffin parts in paraffin blocks; Microtome sectioning with histopathological sections with a thickness of 3-5 mm; coloring and installation.

As coloring methods, it was used: hematoxylin-eosin (H.E.) and Masson tricyclic with aniline blue. The images of the histopathological analysis were taken in bright field microscopy using Leica DM750 microscope with ICC50 HD camera (MEDIST Life Science, Bucharest, Romania).

\section{Results}

\subsection{Post Exploratory Evaluation of Stents' Surface by Light Microscopy}

Thirty-five stents were extracted by autopsy so that they could be further analyzed. Tissue was removed from the recovered stents. Residual tissues were removed by incubating the stent in $\mathrm{KOH} 1-\mathrm{M}$ solution, according to ASTM-F561 [31]. The treatment was performed for 7 days observing progressive tissue detachment from the implant as can be seen on the light microscopy from Figure 2.
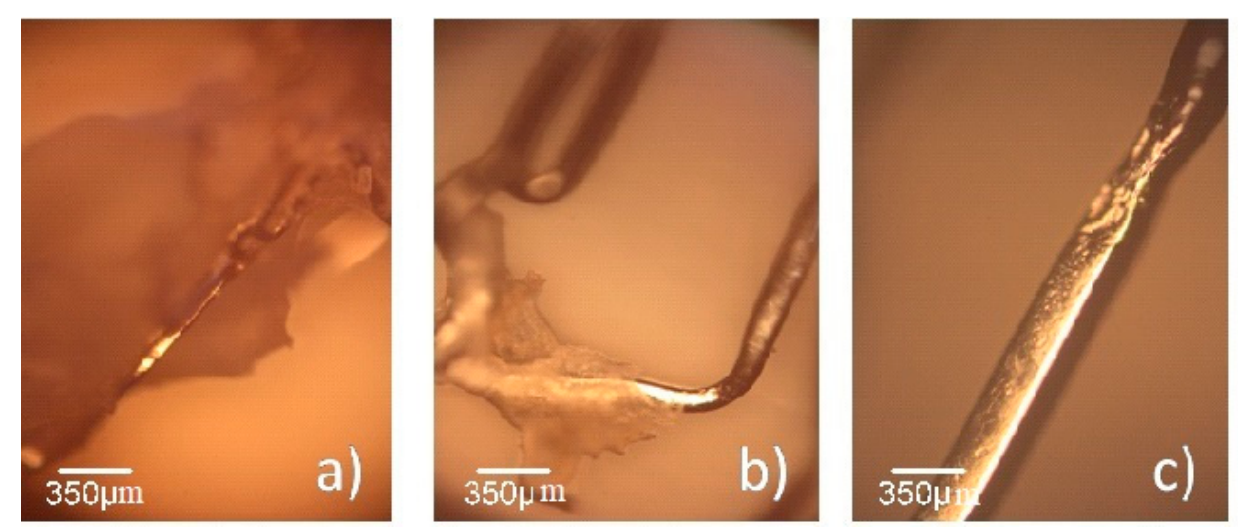

Figure 2. Light microscopy images with the detachment of tissue from stent at various times intervals. (a) After 1 day; (b) after three days; (c) after seven days.

After 7 days of immersion in $\mathrm{KOH}$ solution the stents were analyzed by scanning electron microscopy (SEM). Figure 3a-d presents light microscopy images recorded for samples P1, P7, P10, P11 and Figure 3e-scanning electron microscopy image of sample P11 morphology with pitting corroded areas marked by arrows. 

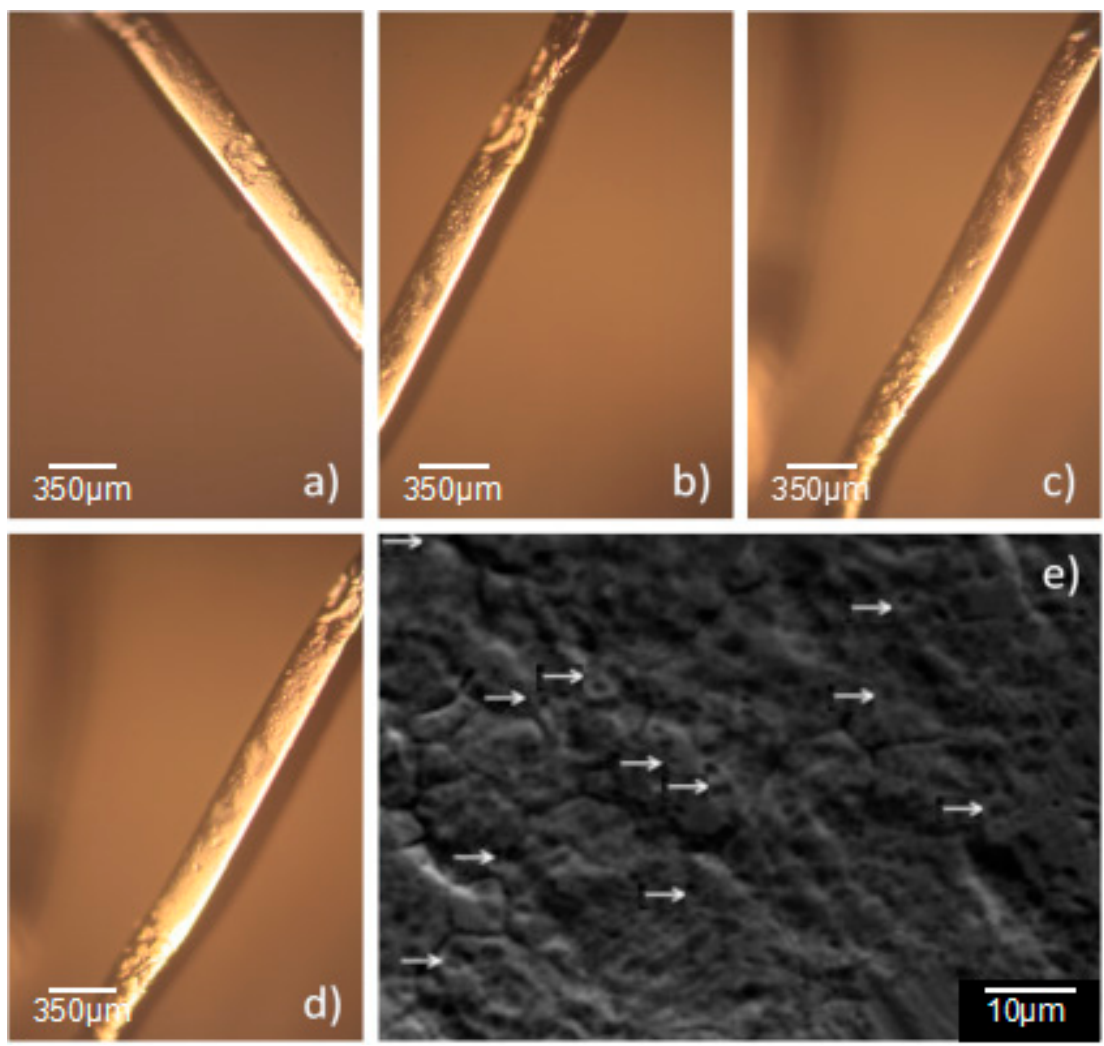

Figure 3. (a-d) Light microscopy images recorded for sample. (a) P1; (b) P7; (c) P10; (d) P11; (e) scanning electron microscopy image of samples P11 morphology with pitting corroded areas marked by arrows.

EDS spectrum recorded for sample P3, that sustains the alloy composition is represented in Figure 4. The EDX analysis of the 35 stents, small variations of the two main elements, Co and Cr, were observed. These deviations are within the standard deviations accepted by the manufacturer. However, for 21 of the 35 stents analyzed, the same chemical composition was obtained, the one presented in the materials chapter. These 21 recovered stents with the same chemical composition were further tested to this work.

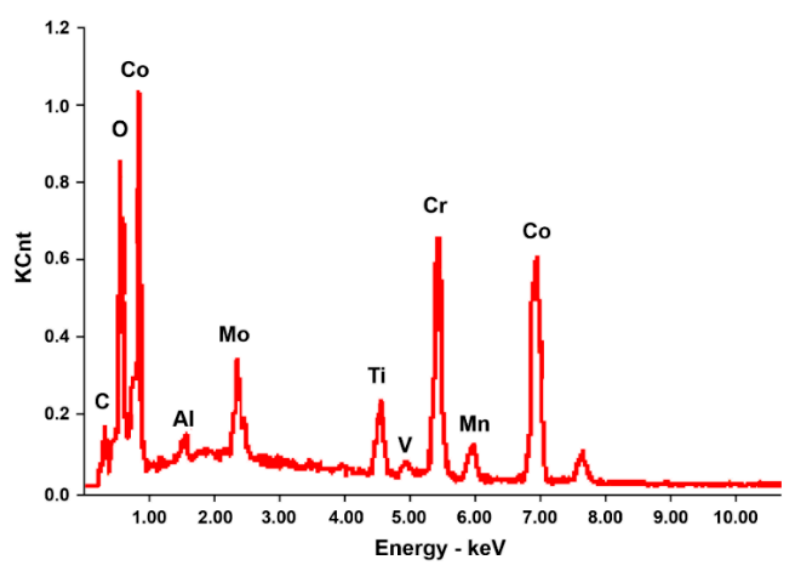

Figure 4. X-ray energy spectrum for the elemental analysis of sample P3.

In order to observe the influence of $\mathrm{KOH}$ solution on the recovered $\mathrm{CoCr}$ stent's surfaces, a new stent with an identical composition was submerged in the same type of solution for 7 days and used as control. The SEM images of the control stent before and after 7 days of immersion in $\mathrm{KOH}$ solution are represented in Figure 5a,b, respectively. 

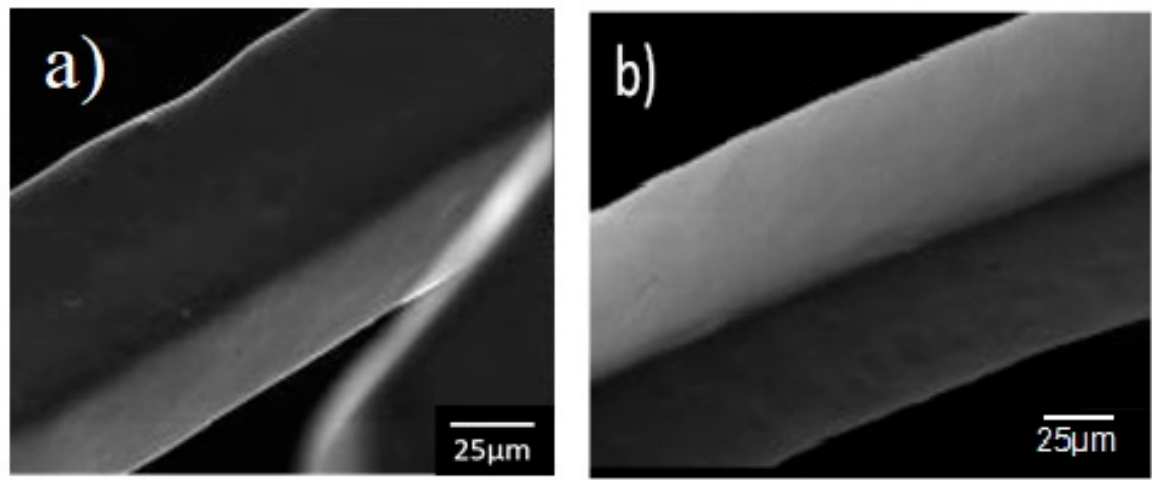

Figure 5. Scanning electron micrography of control stent (a) before and (b) after 7 days of immersion in $\mathrm{KOH}$ solution.

\subsection{ICP-MS Determination}

ICP-MS analysis was performed for all 21 stents recovered from the autopsy with the same chemical composition. For $\mathrm{Co}^{2+}, \mathrm{Cr}^{2+}, \mathrm{Mo}^{2+}$ and $\mathrm{Ti}^{4+}$ ions release determination an analysis of $\mathrm{KOH}$ solution in which it was immerged the control stent highlighted quantities of 6 ppb metallic ions cumulated, considerable negligible and which was related to their natural presence or background noise of ICP-MS device were done. Tissues' digestion recovered from the stents was done by immersing them in concentrated $\mathrm{HNO}_{3}$, acid digestion being done over 3 days.

ICP-MS analysis of digested tissue showed the presence of metallic ions and it is represented for all 21 samples in Figure 6.

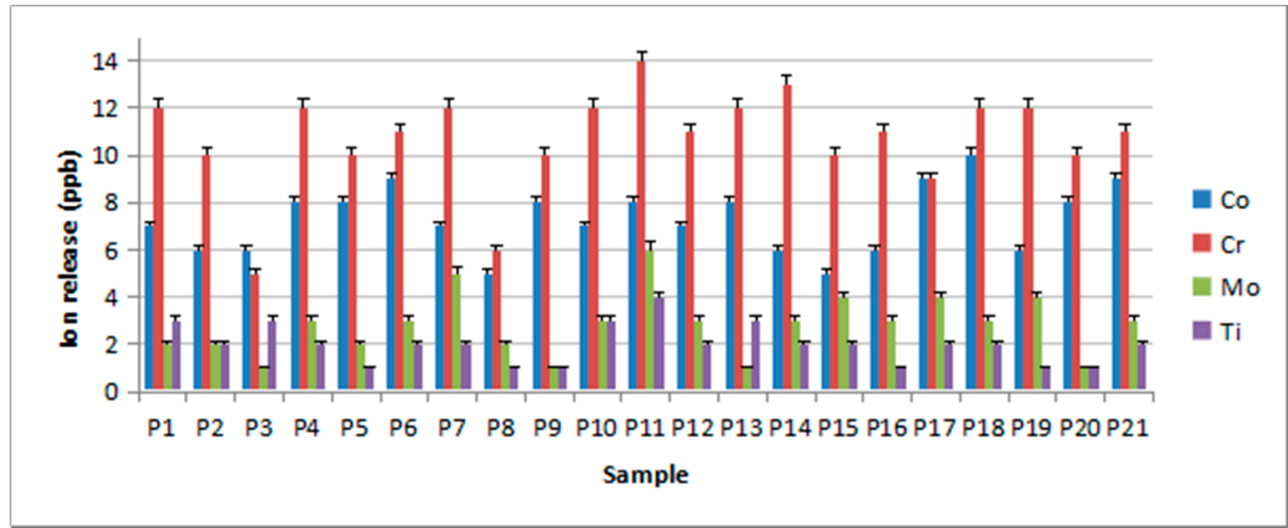

Figure 6. Ion release of $\mathrm{Co}, \mathrm{Cr}$, Mo and $\mathrm{Ti}(\mathrm{ppb})$ from stents by inductively coupled plasma mass spectrometry (ICP-MS) techniques.

The quantities of $\mathrm{Cr}$ ions detected in the tissues were between $5 \mathrm{ppb}(\mathrm{P} 3)$ and $14 \mathrm{ppb}(\mathrm{P} 11)$ and for Co ions were found ranges of $5 \mathrm{ppb}$ (P15) and $10 \mathrm{ppb}$ (P18). There were also detected small quantities of Mo and Ti. These quantities are between 1 ppb (P5) and 6 ppb (P11) for Mo and between 1 ppb (P5, P8, P9, P16, P19, P20) and 4 ppb (P11).

In addition, in all tested tissues was found iron, but this was not reported because it cannot be differentiated between the iron coming from the stent or the one present in the blood. For the other elements in the stent composition ( $\mathrm{Al}, \mathrm{V}, \mathrm{Mn}$ ), which are in very small percentages present in the alloy from which the stent is made, no quantities of metal ions released in the adjacent tissue were determined due to the fact that these metallic ions released were probably below the detection limit of the ICP-MS device which is $0.001 \mathrm{ppb}$.

Increased levels of metallic elements were measured in tissue surrounding corroded stents (P1, P11, P14, P18), whereas negligible levels where measured in tissue surrounding unaltered stents 
(P3, P8). Due to the accentuated degradation of the samples P1, P11, P14, P18 in the tissues surrounding the respective stents, higher amounts of $\mathrm{Co}$ and $\mathrm{Cr}$ ions were highlighted compared to the other samples P3, P8.

In addition, samples P4, P4, P7, P10, P13 presents increased levels of metallic elements that were measured in tissue surrounding corroded stents.

SEM analysis supports the results of ICP-MS showing that there are defects on the surface due to various forms of corrosion. Figure 7 shows that on the surface of the P14 sample there are pits of irregular shapes and different sizes. Occasionally crack lines with approximate dimensions could be observed (depth $=3 \mu \mathrm{m}$, width $=\sim 10 \mu \mathrm{m}$ ) that formed perpendicular to the direction of the stent strut.
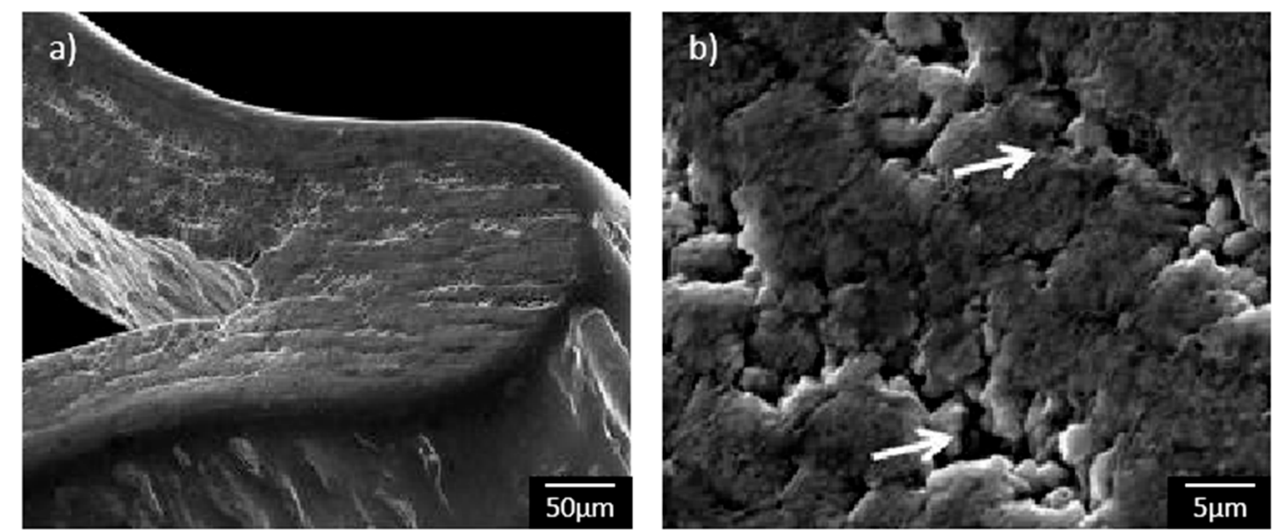

Figure 7. SEM micrographs pictures for P14 showing the presence of pits on the surface (arrows indicate pits); Magnification; (a) 400x; (b) 4000x.

SEM images for samples P11 (Figure 8a) and P1 (Figure 8b) showed the presence of pits on the stent surface, indicated by arrows.
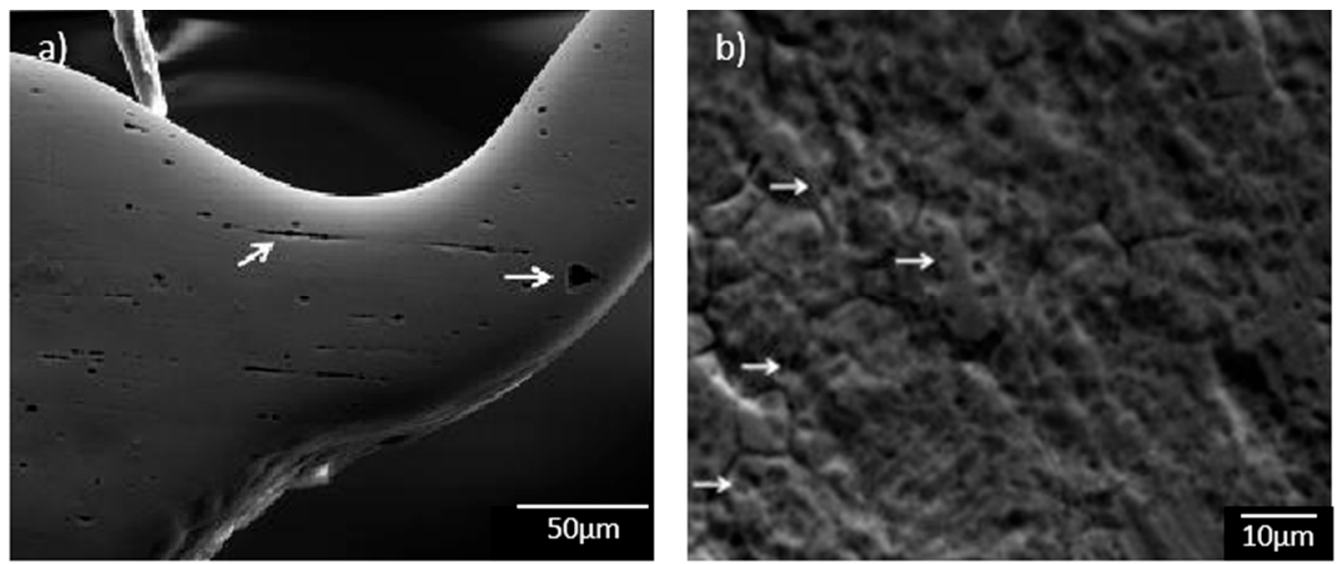

Figure 8. SEM images for (a) P11 sample at 400× magnification; (b) P1 sample at 4000× magnification.

For samples P3 and P8, the SEM analysis showed that the stent surfaces did not show changes as can be seen in Figure 9. 

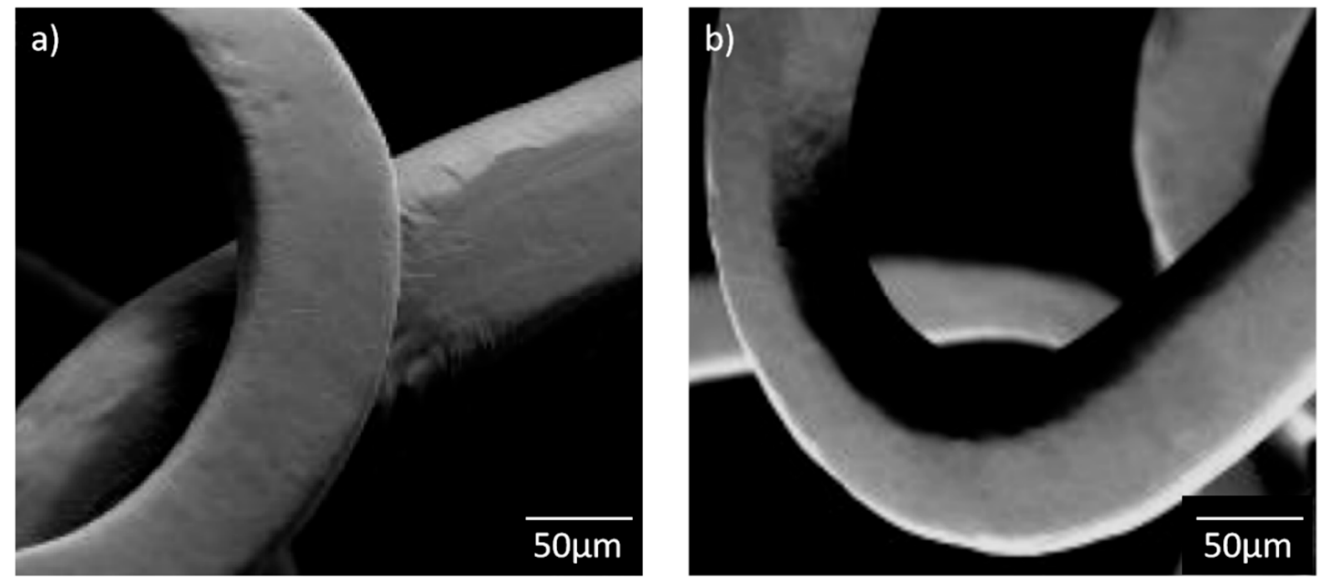

Figure 9. SEM images for (a) P3; (b) P8 sample; both samples 400× magnification.

The P11 specimen was recovered on the INML Bucharest during autopsy on a 66-year-old man after unresponsive cardiopulmonary arrest because of multisystem organ failure (MSOF). He was known with atherosclerosis and atheromatous disease on all 3 coronary arteries with percutaneous coronary intervention (PCI) on right coronary artery (RCA) with bare stent after inferior-posterior myocardial infarction and percutaneous transluminal coronary angioplasty (PTCA) with double pharmacology active stents on anterior interventricular artery (IVA) and left circumflex coronary artery (LCX), terminal kidney failure, severe high blood pressure, chronic obstructive pulmonary disease (COPD), anemia and severe cholestasis.

The P14 sample was recovered from a 56-year-old woman with a history of coronary artery disease and posterior myocardial infarction. She underwent one stent placements in the RCA and four stents in the left anterior descending (LAD) coronary artery along the length of the vessel.

The sample P18 - belonging of a 48-year-old man with hypertension and coronary artery disease. The patient was subjected to a percutaneous coronary intervention with placement of one stent in the RCA and other in the LAD.

P3 - 65-year-old woman with a history of Type II diabetes developed an anterior myocardial infarction. Left heart catheterization revealed an occluded LAD. The percutaneous coronary intervention was performed with placement of bare metal sent. The patient was given inotropes and her medical condition was improved but returned to the hospital with congestive heart failure leading to death. At autopsy, a diffuse calcification surrounding stent was observed.

The presence of metals in the tissue from around explanted stent was also confirmed by TEM method. The tissue was dissolved and then was centrifuged. The resulting precipitate was visualized by TEM (Figure 10).

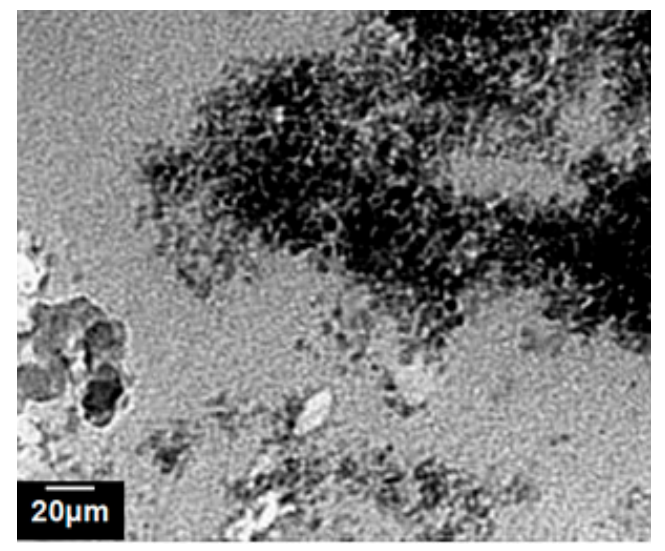

Figure 10. Transmission electron microscopy images of sample P11 morphology. 


\subsection{Evaluation of Tissue/Stents Interface by Histopathological Analysis of Surrounding Tissue}

Samples used in histopathological analyses are taken downstream/upstream of samples used in ICP-MS analysis.

From the 21 analyzed samples, approximately 43\% showed cases of restenosis. The most relevant cases are presented below.

Radiography of one sample (P11) showed diffuse calcification surrounding a stent (Figure 11a) and associated cross section show a reendothelization of the stent struts with absence of in-stent restenosis (ISR) (Figure 11b).
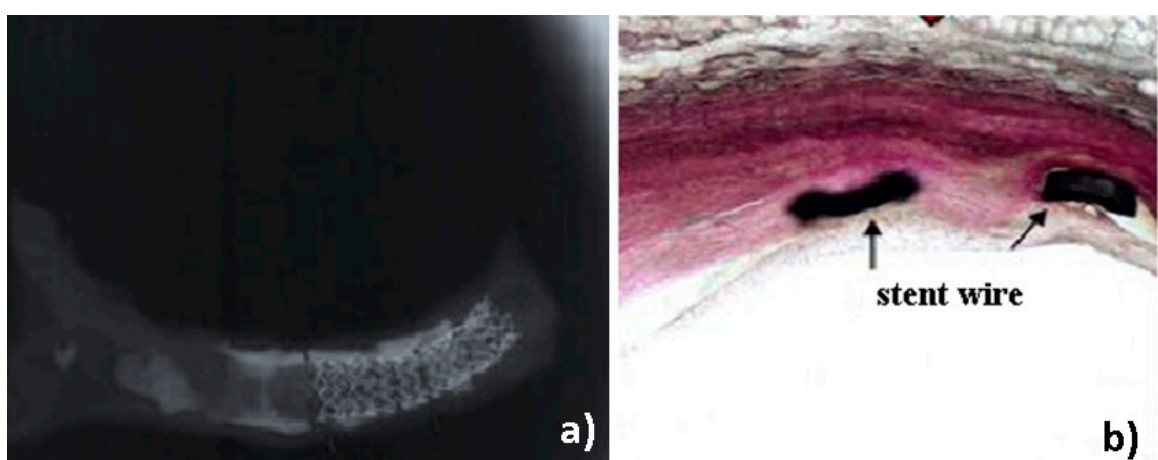

Figure 11. (a) Radiography and (b) associated cross section of sample P11.

The tissue samples were subjected to anatomopathological investigations according to the protocols mentioned in the subchapter Materials and Methods. For upstream sample P11, the following images presented in Figure 12 were obtained.



Figure 12. Rigid ecaudate arteriosum (concentric and predominantly left) fibrosis and calcifications (marked by arrow) that have surrounded one of the stents taken; the polarized light exam did not reveal fragments from the stent in peripheral tissue; hematoxylin-eosin staining; magnification $\times 200$.

The wall of the blood vessel can be observed in Figure 13. In Figure 14 we can see hyperemic capillaries (red blood cells are agglutinated due to hypoxia and yellow), inflammatory infiltration with diffuse lympho-monocytes and macrophages (top center), from a recent infarction (right center) in the regeneration area (approximately 7 days after ischemia); noteworthy conjunctive fibers, corrugated, red (old response to hypoxic aggression). 




Figure 13. Plaque of calcified atheroma (marked by arrows); the take-off is from the stent vessel wall upstream of it; hematoxylin-eosin staining; magnification $\times 200$.

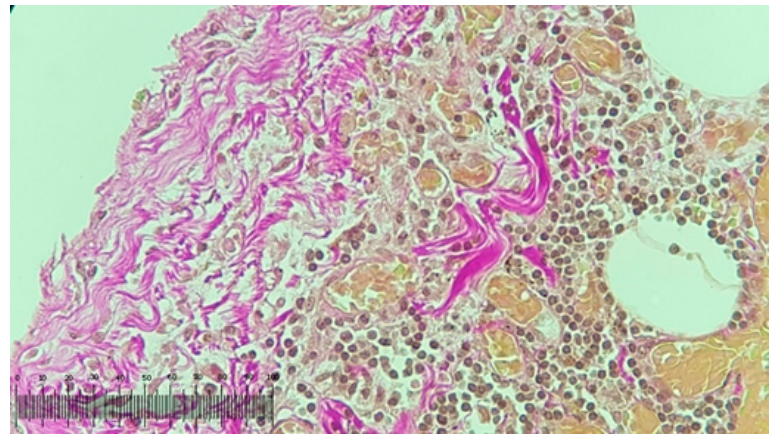

Figure 14. Inflammatory infiltration with diffuse lympho-monocytes and macrophages, 7 days after ischemia.

For sample P14 a cross section of coronary artery (Figure 15a) indicates the concentric narrowing of coronary lumen by the proliferation of endothelial cells approximately $70 \%(\mathrm{~N})$. There is also an area of calcification outside the sent-possibly the old calcified atheroma plaque (C) that produced the initial stenosis of the artery. In the cross section, the absence of metal turns in the stents is observed in the area marked with *, possibly due to fracturing processes following the pressure exerted by the calcified area.

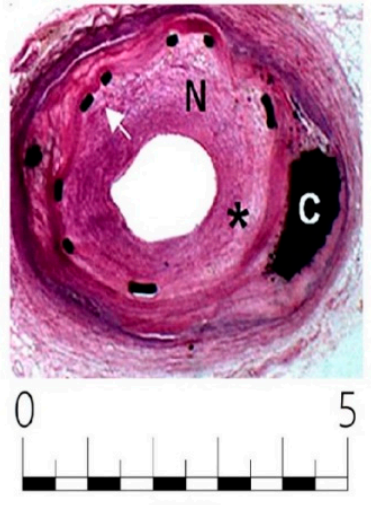

$\mathbf{a}$

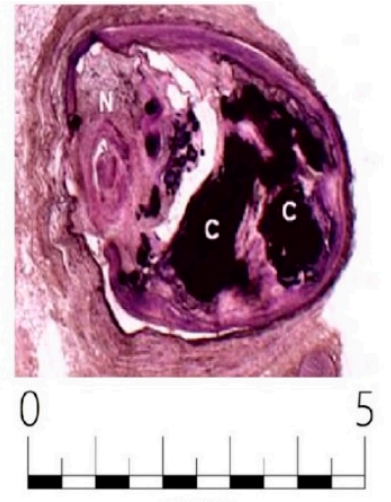

b

Figure 15. Cross section of RCA for: (a) sample P14 showing 70\% ISR (N) and calcified atheroma plaque $(\mathrm{C})$ can be observed. Arrows show stent struts and the absence of stent struts is indicated by the asterisk $\left(^{*}\right)$; (b) sample P18 with peripheral calcifications $(\mathrm{C})$ compressed behind the stent threads and the arterial lumen almost completely restenosed $(\mathrm{N})$. 
For sample P18 (Figure 15b) the sectioned tissue shows compressed peripheral calcifications (C) behind the stent threads and the almost completely restenosis of the arterial lumen $(\mathrm{N})$.

\section{Discussion}

All 21 stents recovered from which the tissue was removed presented various degrees of surface corrosion. Stents' surface present non-corroded areas-but also pitting corroded areas (pitting corroded areas marked by arrow) —as can be observed in Figure 3.

EDS analysis revealed that 21 stents had the same chemical composition. The EDS spectra support highlighted the stent composition as being Co 50.5\%, Cr 24.2\%, Mo 6\%, Mn 0.3\%, V 0.45\%, Ti 4.82\%, C $2.2 \%$, O $11.1 \%$ and $\mathrm{Al} 0.7 \%$.

After exposing to $\mathrm{KOH}$ solution, the control stent surface was uniform and unmodified. This indicates that there was no microtopographic notable effect from the $\mathrm{KOH}$ solution over the control stent's surface.

The heavy metal levels observed in this study due to corrosion were very low. According to the literature, the main cause of ion release on intravascular $\mathrm{CoCr}$ stents is the design consisting of many curves. The decrease of anticorrosion properties due to the sharp and high curve angle which cause gaps between the stents were observed [32]. It is known that very low concentrations of cobalt cause significant expression of endothelial cell adhesion molecule as well as adhesion of polymorphonuclear neutrophil granulocytes to endothelial cells in vitro [33]. Because chromium ions can be triggered by chemical dissolution, which is enhanced in protein containing media, the amount of ions released must be carefully monitored [34,35]. Small chromium ions quantities are nontoxic, but a high concentration into human body may induce carcinogenic risk [36]. In our study, a very small amount of chromium ions released was detected. TEM image of sample P11 show an agglomeration of particles that can reach us to a few micrometry. Agglomeration constituent particles have nanometric dimension and these particles being able to form metallic complexes which can influence local cellular function involved in process of restenosis.

The used old metallic stents were associated with in-stent restenosis (ISR), a multifactorial process consisting of inflammation and non-intimal tissue hyperplasia. Under these conditions, the investigation of the inflammatory potential of the detached metal particles (ion release), from the stents in adjacent tissues it had to be performed.

The images obtained for the wall of the blood vessel show the calcium calcified plate with micro calcifications in the periphery.

Following the anatomopathological investigations, we can say that the image obtained for downstream sample P18 is infiltrated with diffuse lympho-monocytes and macrophages, hyperemic capillaries from the regeneration area of a recent infarction.

Inflammation is associated with development of ISR. It is well known that metallic ions modulate biologic responses including inflammation, characterized by infiltration of macrophages and other inflammatory cells at metallic implants sites [37]. According with our ICP-MS analysis, P18 sample present a high level of $\mathrm{Co}$ and $\mathrm{Cr}$ ions release compared with the other analyzed samples.

The metallic ion release is capable to activate macrophages to produce cytokines and active metalloproteinase and therefore generates and active microenvironment around the corroded stents possibly leading to ISR.

\section{Conclusions}

Based on experimental data, a design research for tissue removal from the recovered metallic stents during post-mortem autopsy and for stents characterization was developed. Recovered stents were characterized by surface morphology techniques like light microscopy (LM), scanning electron microscopy with energy dispersion spectra module (SEM-EDS) and electronic transmission electron microscopy (TEM). The presence of metals in the tissue from around explanted stent was also confirmed by TEM method. The released metal ions were measured using inductively coupled plasma mass 
spectrometry (ICP-MS) establishing low metals level. Histopathological analysis was used to highlight host tissue response to the implanted stents and calcifications on the stents surface. The inflammatory tissue responses were identified and marked. More experiments with the same design research from other metallic stents could be a help for the interventional cardiologist in the future.

Author Contributions: Conceptualization, D.I., F.G. and G.-R.P.-D.; methodology, G.-R.P.-D. and M.P.; validation, M.P., I.D. and M.S.; formal analysis, F.G., D.I. and M.P.; investigation, G.-R.P.-D. and M.S.; writing-original draft preparation, D.I., F.G. and M.P.; writing-review and editing, I.D., D.I. and F.G.; supervision, I.D. and M.S. All authors have read and agreed to the published version of the manuscript.

Funding: This research received no external funding.

Conflicts of Interest: The authors declare no conflict of interest.

\section{References}

1. Jang, S.H.; Lee, D.H.; Ha, J.Y.; Hanawa, T.; Kim, K.H.; Kwon, T.Y. Preliminary evaluation of mechanical properties of Co-Cr alloys fabricated by three new manufacturing processes. Int. J. Prosthodont. 2015, 28, 396-398.

2. Andrei, M.; Galateanu, B.; Hudita, A.; Costache, M.; Osiceanu, P.; Moreno, J.M.C.; Demetrescu, I. Electrochemical comparison and biological performance of a new CoCrNbMoZr alloy with commercial CoCrMo alloy. Mater. Sci. Eng. C 2016, 59, 346-355.

3. Song, S.H.; Min, B.K.; Hong, M.H.; Kwon, T.Y. Application of a novel CVD TiN coating on a biomedical Co-Cr Alloy: An evaluation of coating layer and substrate characteristics. Materials 2020, 13, 1145.

4. Romonti, D.C.; Iskra, J.; Bele, M.; Demetrescu, I.; Milošev, I. Elaboration and characterization of fluorohydroxyapatite and fluoroapatite sol-gel coatings on CoCrMo alloy. J. Alloys Compd. 2016, 665, 355-364.

5. Ionita, D.; Man, I.; Demetrescu, I. The behaviour of electrochemical deposition of phosphate coating on CoCr bio alloys. Key Eng. Mater. 2007, 330, 545-548.

6. Palmaz, J.C. Intravascular stent in the last and the next 10 Years. J. Endovasc. Ther. 2004, 11, 200-206.

7. He, D.; Liu, W.; Zhang, T. The development of carotid stent material. Interv. Neurol. 2015, 3, 67-77.

8. Ionita, D.; Golgovici, F.; Demetrescu, I.; Sajin, M.; Pandelea-Dobrovicescu, G.-R. Effect of human albumin on corrosion and biological behavior of CoCrMo. Mater. Corr. 2017, 68, 876-882.

9. Fukuhara, Y.; Kyuzo, M.; Tsutsumi, Y.; Nagai, A.; Chen, P.; Hanawa, T. Phospholipid polymer electrodeposited on titanium inhibits platelet adhesion. J. Biomed. Mater. Res. Part. B Appl. Biomater. 2016, 104, 554-560.

10. Mindroiu, M.; Cicek, E.; Miculescu, F.; Demetrescu, I. The influence of thermal oxidation treatment on the electrochemical stability of TiAlV and TiAlFe alloys and their potential application as biomaterials. Rev. Chim. 2007, 58, 898-903.

11. Golgovici, F.; Prodana, M.; Pandelea-Dobrovicescu, G.-R. About electrochemical stability of a CoCr alloy used as stents material in medicine. Sci. Bull. B Chem. Mater. Sci. UPB 2018, 80, 219-230.

12. Popa, M.V.; Vasilescu, E.; Drob, P.; Demetrescu, I.; Popescu, B.; Ionescu, D.; Vasilescu, C. In vitro assessment and monitoring of the implant titanium materials-physiological environment interactions. Mater. Corr. 2003, 54, 215-221.

13. Popa, M.V.; Demetrescu, I.; Iordachescu, D.; Cimpean, A.; Vasilescu, E.; Drob, P.; Vasilescu, C.; Istratescu, M. The relation between electrochemical tests and in vitro evaluation of titanium alloy biocompatibility. Mater. Corr. 2007, 58, 687-695.

14. Shabalovskaya, S.; Anderegg, J.; Van Humbeeck, J. Critical overview of nitinol surfaces and their modifications for medical applications. Acta Biomater. 2008, 4, 447-467.

15. Feng, C.C.; Li, L.; Zhang, C.S.; Zheng, G.M.; Bai, X.; Niu, Z.W. Surface characteristics and hydrophobicity of Ni-Ti Alloy through magnetic mixed electrical discharge machining. Materials 2019, 12, 388.

16. Wang, Q.; Fang, G.; Zhao, Y.-H.; Zhou, J. Improvement of mechanical performance of bioresorbable magnesium alloy coronary artery stents through stent pattern redesign. Appl. Sci. 2018, 8, 2461.

17. Lu, P.; Fan, H.; Liu, Y.; Cao, L.; Wu, X.; Xu, X. Controllable biodegradability, drug release behavior and hemocompatibility of PTX-eluting magnesium stents. Colloids Surf. B Biointerfaces 2011, 83, $23-28$.

18. Waksman, R. Update on bioabsorbable stents: From bench to clinical. J. Interv. Cardiol. 2006, 19, 414-421. 
19. Beshchasna, N.; Saqib, M.; Kraskiewicz, H.; Wasyluk, L.; Kuzmin, O.; Duta, O.C.; Ficai, D.; Ghizdavet, Z.; Marin, A.; Ficai, A.; et al. Recent advances in manufacturing innovative stents. Pharmaceutics 2020, 12, 349.

20. Scheinert, D.; Scheinert, S.; Sax, J.; Piorkowski, C.; Braunlich, S.; Ulrich, M.; Biamino, G.; Schmidt, A. Prevalence and clinical impact of stent fractures after femoropopliteal stenting. J. Am. Coll. Cardiol. 2005, 45, 312-315.

21. Huang, K.-H.; Yin, W.-H.; Lee, Y.-T.; Tsao, T.-P.; Lee, K.-C.; Hsiung, M.-C.; Wei, J. Quantification of stent creep by three-dimensional transesophageal echocardiography in patients undergoing transcatheter aortic valve-in-valve implantation for failed bioprostheses. Acta Cardiol. Sin. 2019, 35, 380-386.

22. Paprottka, K.; Paprottka, P.; Reiser, M.; Waggershauser, T. Comparative study of the corrosion behavior of peripheral stents in an accelerated corrosion model: Experimental in vitro study of 28 metallic vascular endoprostheses. Diagn IntervRadiol. 2015, 21, 403-409.

23. Maron, B. Surgery for Hypertrophic Obstructive Cardiomyopathy alive and quite well. Circulation 2005, 11, 2016-2018.

24. Rabbat, M.G.; Bavry, A.A.; Bhatt, D.L.; Ellis, S.G. Understanding and minimizing late thrombosis of drug-eluting stents. Cleve Clin. J. Med. 2007, 74, 129-136.

25. Hou, R.; Wu, L.; Wang, J.; Yang, Z.; Tu, Q.; Zhang, X.; Huang, N. Surface-degradable drug-eluting stent with anticoagulation, antiproliferation, and endothelialization functions. Biomolecules 2019, 9, 69.

26. Lu, J.; Yao, C.; Yang, L.; Webster, T.J. Decreased platelet adhesion and enhanced endothelial cell functions on nano and submicron-rough titanium stents. Tissue Eng. A 2012, 18, 1389-1398.

27. Nagai, A.; Suzuki, Y.; Tsutsumi, Y.; Nozaki, K.; Wada, N.; Katayama, K.; Hanawa, T.; Yamashit, K. Anodic oxidation of a Co-Ni-Cr-Mo alloyand its inhibitory effect on platelet activation. J. Biomed. Mater. Res. B. Appl. Biomater. 2014, 102, 659-666.

28. ASTM F-86. Standard practice for surface preparation and marking of metallic surgical implants. In Annual Book of ASTM Standards: Medical Devices and Services Philadelphia: American Society for Testing and Materials; ASTM F-86: Philadelphia, PA, USA, 1995.

29. Demetrescu, I.; Luca, D.; Ionita, D.; Prodana, M. Evaluating environmental impact factors as heavy metals on behaviour of temporary teeth. Mol. Cryst. Liq. Cryst. 2010, 523, 73-81.

30. Totea, G.; Ioniţă, D.; Demetrescu, I. ICP-MS determinations in sustaining corrosion data of 316 stainless steels in bioliquids. Sci. Bull. B Chem. Mater. Sci. UPB 2014, 76, 57-66.

31. ASTM-F561. Annual Book of ASTM Standards: Medical Devices and Services. Philadelphia: American Society for Testing and Materials; ASTM-F561: Philadelphia, PA, USA, 2002; pp. 97-101.

32. Kapnisis, K. Multi-scale mechanical investigation of stainless steel and cobalt chromium stents. J. Mech. Behav. Biomed. 2014, 40, 240-251.

33. Wagner, M.; Klein, C.L.; Van Kooten, T.G.; Kirkpatrick, C.J. Mechanisms of cell activation by heavy metal ions. J. Biomed. Mater. Res. 1998, 42, 443-452.

34. Lundin, M.; Hedberg, Y.; Jiang, T.; Herting, G.; Wang, X.; Thormann, E.; Blomberg, E.; Odnevall Wallinder, I. Adsorption and protein-induced metal release from chromium metal and stainless steel. J. Colloid. Interface Sci. 2012, 366, 155-164.

35. Kocijan, A.; Conradi, M.; Schon, P.M. Austenitic and duplex stainless steels in simulated physiological solution characterized by electrochemical and X-ray photoelectron spectroscopy studies. J. Biomed. Mater. Res. B 2012, 100B, 799-807.

36. Wilbur, S.; Abadin, H.; Fay, M.; Yu, D.; Tencza, B.; Ingerman, L.; Klotzbach, J.; James, S. Toxicological Profile for Chromium; Toxicological Profiles; Agency for Toxic Substances and Disease Registry (ATSDR): Atlanta, GA, USA, 2012.

37. Santin, M.; Mikhalovska, L.; Lloyd, A.W.; Mikhalovsky, S.; Sigfrid, L.; Denyer, S.P.; Field, S.; Teer, D. In vitro host response assessment of biomaterials for cardiovascular stent manufacture. J. Mater. Sci. Mater. Med. 2004, 15, 473-477.

(C) 2020 by the authors. Licensee MDPI, Basel, Switzerland. This article is an open access article distributed under the terms and conditions of the Creative Commons Attribution (CC BY) license (http://creativecommons.org/licenses/by/4.0/). 